\title{
Role of fiberoptic bronchoscopy in patients with respiratory complications in multispecialty intensive care units
}

\author{
S. Harikrishnan ${ }^{1, *}$, A. Sundarajaperumal ${ }^{2}$, Nagarajan ${ }^{3}$ \\ ${ }^{1}$ Assistant Professor, ${ }^{3}$ Senior Resident, Dept. of Respiratory Medicine, Velammal Medical College, Madurai, Tamil Nadu, \\ ${ }^{2}$ Associate Professor, Dept. of Respiratory Medicine, Madras Medical College, Chennai, Tamil Nadu, India
}

*Corresponding Author:

Email: medicokrish@gmail.com

\begin{abstract}
Introduction: To determine the contribution and safety profile of flexible fiberoptic bronchoscopy in intensive care units in terms of therapeutic outcome in lung collapse patients.

Methodology: Patients with respiratory complications from various speciality intensive care units were enrolled. After clinical and radiological evaluation, if conservative management fails, bronchoscopic intervention was considered. Bronchoscopy was performed according to British Thoracic Society guidelines after informed consent. Swivel-T-Tube adapter was used for the patients in mechanical ventilation. Data such as chest radiograph, arterial blood gas, Bacterial culture of tracheal and BAL secretions between before and after brochcoscopy ( $6 \& 24 \mathrm{hrs})$ were analysed using standard SPSS software.

Results: In our study 63 patients were enrolled, Most of the patients were in mechanical ventilation 85\% ( $\mathrm{n}=54 / 63$ ). In our study, the indications for bronchoscopy were suspected atelectasis $57.1 \%(\mathrm{n}=36)$, persistent pulmonary infiltrates $30 \%$ ( $\mathrm{n}=27)$. In suspected atelectasis cases $(n=36)$ bronchoscopic findings were mucous plug in $61 \%(n=22)$, blood clot in $11 \%(n=4)$ and purulent secretions $22 \%(\mathrm{n}=8)$ and mucoid secretions in $5 \%(\mathrm{n}=2)$. Post brochoscopic chest radiograph of suspected ateletasis showed partial resolution in $38.8 \%(n=14)$ and complete resolution in $47.2 \%(n=17)$.

Conclusion: In our study contribution of bronchoscopy played a vital role in therapeutic outcome. Patients had good response to bronchoscopy $85 \%$ improvement when intervened within 5 days of admission in ICU. No life threatening complications were encountered; hence bronchoscopy is safe in critically ill patients, if basic precautions are taken.
\end{abstract}

Keywords: BAL and VAP; Fiberoptic bronchoscopy in intensive care units; Lung atelectasis in ICU; Safety of FOB in ICU.

\section{Introduction:}

Since fiber-optic bronchoscopy introduction in $20^{\text {th }}$ century by Ikeda, has played an important role in pulmonology [1]. Other than diagnostic use, now it is also implemented for therapeutic purposes in different clinical scenarios [2,3]. Bronchoscope is now used widely not only in stable patients, also in critically ill patients as its versatility and portability allows bronchoscopist to do interventions at bedside. In critically ill patients it is used increasingly because of its diagnostic accuracy and safety $[3,4]$.

\section{Aims and objectives:}

To determine the contribution and safety profile of flexible fiberoptic bronchoscopy in intensive care units in terms of therapeutic outcome in lung collapse patients.

\section{Methodology:}

Call overs from the intensive care units of various specialities were attended and patients were initially evaluated clinically and radiologically. If respiratory complications were diagnosed in the patients, they were started on conservative management. If the conservative management failed to improve the patient's oxygenation or refractory to empirical antibiotics, bronchoscopic intervention was planned. Thus we enrolled consecutive 61 patients called for expert opinion from various speciality intensive care units with respiratory complications refractory to conservative management were needed bronchoscopic intervention during the study period. In which 36 patients had lung collapse with failed chest physiotherapy.

\section{Inclusion criteria:}

a. Patients in multispecialty intensive care units with lung collapse in whom the conservative management failed to improve the pulmonary status.

b. Preferred age of the patient was 18-65 years

\section{Exclusion criteria:}

a. Patients with whose relatives not willing to give the consent

b. Patient with recent myocardial infarction

c. Patients with increased intracranial tension

Study place:

Various multispecialty intensive care units in the Rajiv Gandhi Government General Hospital, Chennai. The speciality ICUs were segregated as follows:-

1. Medical and toxicology intensive care units

2. Surgical intensive care units

3. Neurology / trauma care intensive care units

4. Post-anaesthetic intensive care unit

5. Other specialities which were included were nephrology ICU, vascular surgery ICU, radiation oncology ICU.

Study design: Prospective and observational study.

Study period: January 2015 - October 2015

Material and Methods:

Patients with respiratory complications, who were critically ill in multi speciality intensive care units seen 
in call overs, were examined clinically and radiologically. If conservative management is not given by the referring unit, conservative management was advised initially. On further follow up if patient's pulmonary condition not improved or get worsened they were enrolled in our study. In patients who had adequate conservative management by referring unit and refractory to conservative management were also enrolled during initial evaluation itself. Patients were intervened for following indications.

1. Indication for bronchoscopy in our study:

a. Suspected lung atelectasis $[5,6]$

b. Retained airway secretions $[5,6]$

Calls were received from various speciality ICUs for the favour of management of persistent hypoxia secondary due to lung collapse, retained airway secretions. After initial assessment, conservative managements such as chest physiotherapy in cases of suspected collapse or frequent airway suctioning in cases of increased secretions were advised. Patients were followed up every $12 \mathrm{hrs}$. If there is adequate expansion of the lung with improvement in arterial blood gas analysis, conservative management was continued $[7,8]$. But in cases were conservative management failed to improve the pulmonary status, need of bronchoscopy were taken into account for further management. Some cases were referred after failure of conservative management, in such patients need of bronchoscopy were taken into account during initial evaluation itself. After analysing the general conditions and contraindications for the bronchoscopy by expert pulmonologists flexible bronchoscopy was done in 36 patients for the favour of expansion of lung collapse, Informed and written consent were obtained from the close relatives of the patients.

\section{Observations and Results}

\section{Statistical analysis:}

Categorical data were described using frequencies and percentages, while quantitative data was described using mean $\pm \mathrm{SD}$ or median (IQR) depending on the type of data. Cross-tabulations were done for important variables. Association was determined using chi-square test or McNemar test. Statistical significance was determined at $5 \%$.

Observation: 61 patients with respiratory complications refractory to conservative managements were intervened and their data were analysed for contribution of flexible fiber-optic bronchoscopy in intensive care unit.

Age: Mean age observed were $45 \pm 12$ (mean \pm SD).

In patient with trauma mean age was $43.5(\mathrm{n}=21)$, in which 12 patients had bronchoscopy for lung atelectasis. Only two patients above 45 were did not responded to bronchoscopic intervention within 24 hours.

Sex: In total of 61 patients enrolled, 44 were male $69.8 \%$ and 19 were females $30.2 \%$.

Initial chest-X ray findings: In chest radiograph taken during initial evaluation, lung atelectasis was made out in 36 patients in which one patient was diagnosed as total lung collapse whose primary diagnosis was road traffic accident - poly trauma with head injury. 29 patients out of 36 were lobar collapse and 6 were multilobar collapse. 25 patient's chest radiograph showed airspace opacities in which 19 patients had bilateral lesion and 6 had unilateral lesion.

๑LEFT $\square$ RIGHT $\square$ BILATERAL

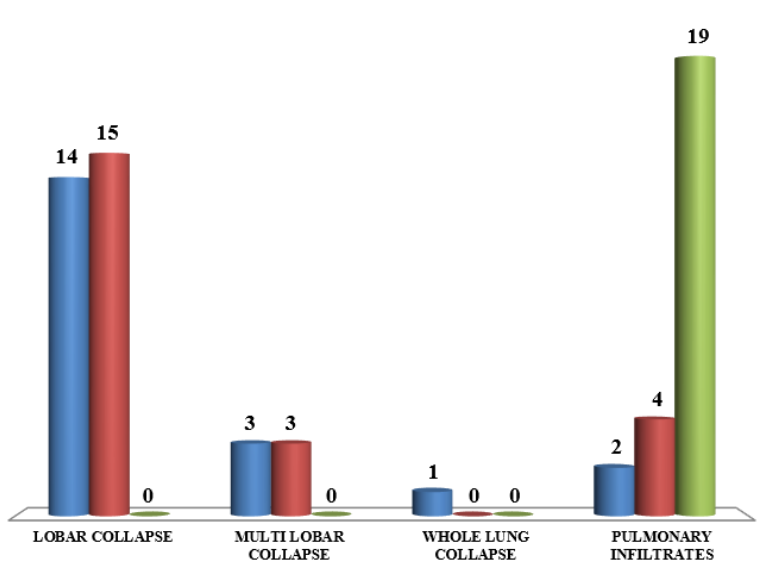

Fig. 1: Shows initial chest radiograph findings

Table 1: Shows initial chest radiograph findings

\begin{tabular}{|l|c|c|}
\hline Initial Chest Radiograph Findings & $\mathbf{n}$ & \% \\
\hline Lobar Collapse & 29 & 46 \\
\hline Multi-Lobar Collapse & 6 & 9.5 \\
\hline Whole Lung Collapse & 1 & 1.5 \\
\hline Pulmonary Infiltrates & 25 & 39.6 \\
\hline
\end{tabular}

IP Indian Journal of Immunology and Respiratory Medicine, July-September, 2018;3(3):117-120 
Changes in post bronchoscopy chest radiograph after 6 hours in patients with lung atelectasis: Out of 63 patients, 36 patients had for lung atelectasis in which 29 were lobar collapse, 6 were multilobar collapse, 1 was a total lung collapse. When comparing pre bronchoscopy chest radiograph with post bronchoscopy radiograph at $6^{\text {th }}$ hour the following observations were made.

Out of 29 lobar collapse patients, 37.9\% showed partial re-expansion, $37.9 \%$ shows complete re-expansion and $24.1 \%$ showed no expansion.

Table 2: Shows changes in post bronchoscopy chest radiograph after 6 hours in patients with lung atelectasis

\begin{tabular}{|l|c|c|c|c|}
\hline \multirow{2}{*}{$\begin{array}{c}\text { Initial chest } \\
\text { radiograph }\end{array}$} & \multicolumn{2}{|c|}{ Chest radiograph after 6 hours } & Total \\
\cline { 2 - 5 } & $\begin{array}{c}\text { Non } \\
\text { resolution }\end{array}$ & $\begin{array}{c}\text { Partial } \\
\text { re-expansion }\end{array}$ & $\begin{array}{c}\text { Complete } \\
\text { re-expansion }\end{array}$ & \\
\hline Lobar collapse & 7 & 11 & 11 & 29 \\
& $24.1 \%$ & $37.9 \%$ & $37.9 \%$ & $100 \%$ \\
\hline Multi-lobar & 0 & 6 & 0 & 6 \\
collapse & $0 \%$ & $100 \%$ & $0 \%$ & $100 \%$ \\
\hline Whole lung & 0 & 1 & 0 & 1 \\
collapse & $0 \%$ & $100 \%$ & $0 \%$ & $100 \%$ \\
\hline Total & 7 & 18 & 11 & 36 \\
& $19.4 \%$ & $50 \%$ & $30.5 \%$ & $100 \%$ \\
\hline
\end{tabular}

In case of multilobar collapse all patients responded to bronchoscopy at $6^{\text {th }}$ hour itself but no one showed complete re-expansion.

A patient with total lung collapse responded with partial re-expansion at the end of $6^{\text {th }}$ hour.

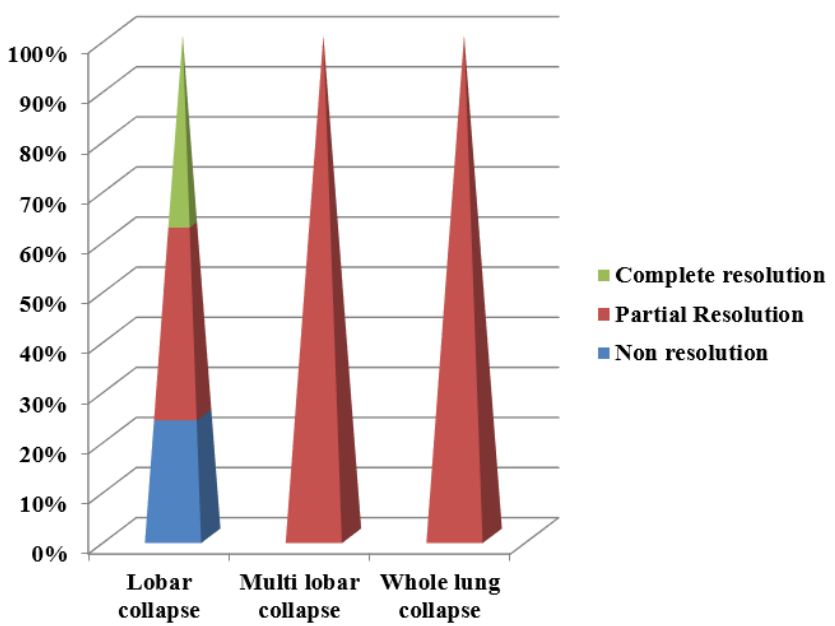

Fig. 2: Shows changes in post bronchoscopy chest radiograph after 6 hours in patients with lung atelectasis

Changes in post bronchoscopy chest radiograph after 24 hours in patients with lung atelectasis: To know the progress of radiological improvement in intervened patients, chest radiograph was repeated after 24 hour. The observations were compared with pre and post bronchoscopy chest radiograph are as follows.

In 29 patients whose chest radiograph showed lobar collapse, 51.7\% had complete re-expansion, $31 \%$ had partial re-expansion. About $17.2 \%$ had no expansion even after $24 \mathrm{hr}$.

Table 3: Shows changes in post bronchoscopy chest radiograph after 24 hours in patients with lung atelectasis

\begin{tabular}{|l|c|c|c|c|}
\hline \multirow{2}{*}{\begin{tabular}{c}
\multirow{2}{*}{$\begin{array}{c}\text { Initial chest } \\
\text { radiograph }\end{array}$} \\
\cline { 2 - 5 }
\end{tabular}} & $\begin{array}{c}|c| \\
\text { Non } \\
\text { resolution }\end{array}$ & $\begin{array}{c}\text { Partial } \\
\text { re-expansion }\end{array}$ & $\begin{array}{c}\text { Complete } \\
\text { re-expansion }\end{array}$ & \\
\hline Lobar collapse & $5(17.2 \%)$ & $9(31 \%)$ & $15(51.7 \%)$ & $29(100 \%)$ \\
\hline Multi-lobar collapse & $0(0 \%)$ & $4(66.7 \%)$ & $2(33.3 \%)$ & $6(100 \%)$ \\
\hline $\begin{array}{l}\text { Whole lung } \\
\text { collapse }\end{array}$ & $0(0 \%)$ & $1(100 \%)$ & $0(0 \%)$ & $1(100 \%)$ \\
\hline Total & $5(13.8 \%)$ & $14(38.8 \%)$ & $17(47.2 \%)$ & $36(100 \%)$ \\
\hline
\end{tabular}


In multilobar collapse, all patients were responded at $6^{\text {th }}$ hour itself by partial re-expansion. After 24 hours it is observed that only 2 patients were further improved to complete re-expansion.

A patient with total lung collapse showed some further improvement in chest radiograph when compared to chest radiograph at $6^{\text {th }}$ hour. But complete re-expansion was not obtained.

Out of 25 patients with persistent airspace opacities, $36 \%(\mathrm{n}=9)$ patients showed minimal resolution of opacity radiologically.

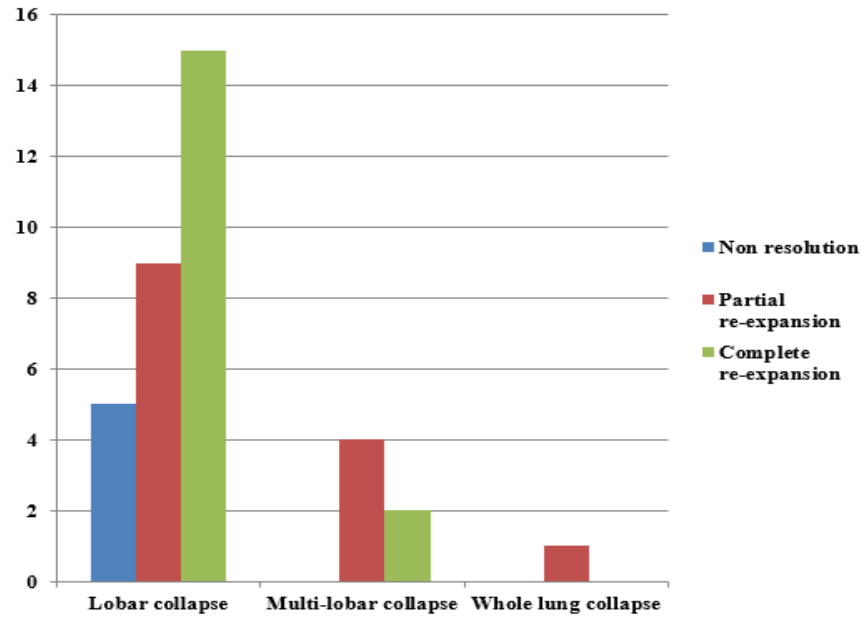

Fig. 4: Shows changes in post bronchoscopy chest radiograph in patients with lung atelectasis

\section{Conclusions:}

About $85 \%$ of patients showed improvement in chest radiograph after bronchoscopy thus plays a vital role in treatment protocol for patients with lung atelectasis in ICU. Most of the patients who needed bronchoscopy were in the mechanical ventilator. Hence patients in mechanical ventilator should be given adequate precautions to prevent respiratory complications. There was about $23 \%$ of difference observed in better therapeutic outcome if intervened in early days of admission in ICU. In our study no major complications were encountered in any of our patients. Hence flexible bronchoscopy is safe in critically ill patients if necessary basic precautions were taken.

Conflicts of interests: None declared Acknowledgements: None

\section{References:}

1. Elsa Guerreiro ds cunha fragoso, MD, and jose Manuel Rosal Goncalves, MD, role of fiberoptic bronchoscopy in intensive care units current practice. J Bronchol Pulmonol 2011;18:69-83.
2. Raoof S, Mehrishi S, Prakash UB. Role of bronchoscopy in modern medical intensive care unit. Clin Chest Med 2001;22:241-61.

3. Estella A, Servicio de Medicina Intensiva, Hospital SAS de Jerez, Jerez de la Frontera, Cádiz Analysis of 208 flexible bronchoscopies performed in an intensive care unit, Spain Med Intensiva 2012;36(6):396-401.

4. Turner JS, Willcox PA, Hayhurst MD, Potgieter PD. Fiberoptic bronchoscopy in the the intensive care unit - a prospective study of 147 procedure in 107 patients. Critical Care Med 1994;22(2):259-64.

5. Dellinger RP. Fiberoptic bronchoscopy in adult airway management. Critical Care Med 1990;18(8):882-87.

6. Albright JM, Davis CS, Bird MD, et al., The acute pulmonary inflammatory response to the graded severity of smoke inhalation injury. Critical Care Med 2012;40(4):1113-21.

7. Bearnis JF, Vernovsky I, Jacobson J. Flexible bronchoscopy in the critical care unit. Chest 2000;118:77.

8. Patel DB, Udwadia ZF. Role of bronchoscopy in an Indian critical care unit an experience of 118 procedures. Thorax 1997;52(6):A65.

How to cite this article: Harikrishnan S., Sundarajaperumal A., Nagarajan. Role of fiberoptic bronchoscopy in patients with respiratory complications in multispecialty intensive care units. $I P$ Indian J Immunol Respiratory Med 2018;3(3):117120. 